\title{
Improvement of the Reliability of a Series-Parallel System Subject to Modified Weibull Distribution with Fuzzy Parameters
}

\author{
Neama Salah Youssef Temraz \\ Mathematics Department, Faculty of Science, Tanta University, Tanta, Egypt
}

Article history

Received: 31-07-2017

Revised: 25-08-2017

Accepted: 31-08-2017

Email: sci_neama55@yahoo.com

\begin{abstract}
This paper introduce a series-parallel system consisted of independent and non-identical components with lifetimes follow the modified Weibull distribution. Reduction method is introduced to improve system reliability. Other methods of hot and cold standby duplication are established to improve system reliability. A procedure for computing the reliability of the original system and the improved systems is presented when the parameters of the modified Weibull distribution become fuzzy. Numerical study is introduced to show the results and compare between different improvement methods.
\end{abstract}

Keywords: Reliability, Series-Parallel System, Fuzzy Numbers, Membership Function, Modified Weibull Distribution, Maximum likelihood Method, Confidence Interval, Cold Standby, Hot Standby, Reduction Method

\section{Introduction}

The reliability of a unit of a system is the probability that the unit will perform its specified function during an interval of time under given conditions. In general, reliability of a system depends on many factors such as the reliabilities of the system units, the configuration of the system, and system failure criteria. In reliability studies, the goal is to predict suitable reliability indices for the system based on component failure data and system design (Hoyland and Rausand, 2004).

In many cases, reliabilities of practical systems should be improved to reach a certain level. Improving the reliability of a system can be achieved by decreasing the failure rate of its components or increasing the repair rate of its repairable components. Improving system reliability can be achieved by other methods such as hot duplication method and cold standby duplication method.

By using reduction method, system reliability is improved by reducing the failure rates of some of its units by a factor $\rho$ where $(0<\rho<1)$. In hot duplication method, some of the system units are duplicated in parallel. In cold duplication method, some of the system units are duplicated by a cold redundant standby component via a switch which can be perfect or imperfect.
Weibull distribution is a flexible distribution that used in system reliability analysis. Weibull distribution can be used to model a variety of life behaviors depending on different values of the parameters. Lai et al. (2003) introduced the modified Weibull distribution as a new lifetime distribution that capable of modeling a bathtub shaped hazard rate function. The proposed model is derived as a limiting case of the Beta Integrated Model and has both the Weibull distribution and Type I extreme value distribution as special cases. The model can be considered as another useful generalization of the Weibull distribution.

Fuzzy set theory was introduced by Zadeh (1965) in order to generalize the theory of classical sets. In a fuzzy set, it is not necessary that the element is a full member of the set or not a member. It can be a partial member of the set. A fuzzy set is defined by a function that maps elements in a domain of concern to their membership value in a set. This function is called the membership function. The most main character of fuzzy set is that membership function gives every element a value of $[0,1]$ as it is grade of membership. The grade of membership has the characteristic that a single value combines the evidence for and the evidence against the element (Novák et al., 1999). 
In Literature, there are many papers that deal with improving the reliability of mathematical models. Khan and Jan (2015) introduced reliability evaluation of a system using modified Weibull distribution. Ezzati and Rasouli (2015) improved system reliability using linearexponential distribution function. El-Damcese (2009) introduced reliability equivalence factors of a seriesparallel system when the system components are independent and identical with a life distribution of Weibull distribution. Reliability equivalence factors for some systems with mixture Weibull failure rates were introduced by Mustafa (2009). Poǵany et al. (2013) improved the reliability of composite system by using reduction method and hot duplication method considering the systems survivor function. Related survivor equivalence functions were derived in all cases when the components lifetime distribution follows the gamma-Weibull distribution.

Since, the exact data about the parameters of a probability distribution cannot be always available so that these parameters can be expressed as fuzzy numbers. These fuzzy numbers can be driven from collected data or the opinions of the experts. El-Damcese and Temraz (2015) proposed analysis of availability and reliability of k-out-of-n model assuming the rates of the model are fuzzy. Cheng and Mon (1993) used the confidence interval for analyzing the fuzzy system reliability. Chen (1994) presented a new method for analyzing the fuzzy system reliability using arithmetic operations of fuzzy numbers. Chen (1996) presented a new method for fuzzy system reliability analysis based on fuzzy time series and the $\alpha$-cuts arithmetic operations of fuzzy numbers.

In this study, the reliability of a series-parallel system consisting of independent and non-identical components is improved assuming that the lifetime of each unit follows modified Weibull distribution. We suppose that the parameters of the modified Weibull distribution are fuzzy numbers with triangular membership functions. Reliability function of the original system and improved systems is derived according to reduction, hot duplication, and cold duplication methods. Finally, we study the model numerically.

This paper is organized as follows. In Section 2, review of modified Weibull distribution is discussed. Maximum likelihood method is introduced to find point estimators for the modified Weibull distribution. Definitions and basics of fuzzy numbers are discussed. In Section 3, the reliability function of the original system is deduced. In Section 4, reduction method is applied to improve the system reliability. In Section 5, cold standby duplication method is presented to increase system reliability. In Section 6, hot standby duplication method is introduced to improve the reliability of the system. In Section 7, a procedure is introduced to show how the intervals for the system reliability are obtained.
In Section 8, a numerical study is presented to illustrate the results and compare between different methods.

\section{Modified Weibull Distribution}

The probability density function of the modified Weibull distribution is defined as:

$$
\begin{aligned}
& f(t)=\beta(\gamma+\lambda t) t^{\gamma-1} e^{\lambda t} e^{-\beta t^{\gamma} e^{\lambda t}} \\
& \beta>0, \gamma \geq 0, \lambda \geq 0, t>0
\end{aligned}
$$

The distribution function is given by:

$F(t)=1-e^{-\beta t^{y} e^{2 t}}$

The hazard rate function is given by:

$h(t)=\beta(\gamma+\lambda t) t^{\gamma-1} e^{\lambda t}$

For $\lambda=0$, we obtain the original Weibull distribution. Different shapes of the probability density and hazard rate functions are illustrated in Fig. 1 and 2.

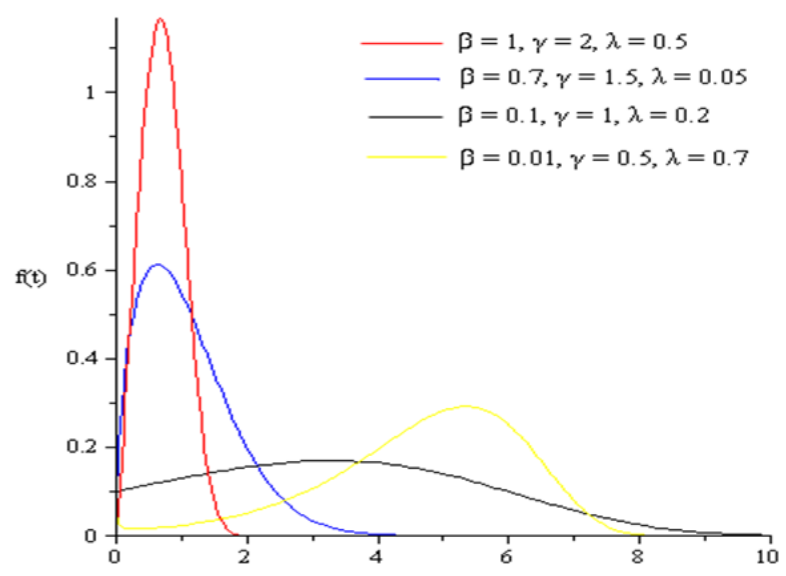

Fig. 1. Different shapes of PDF versus time

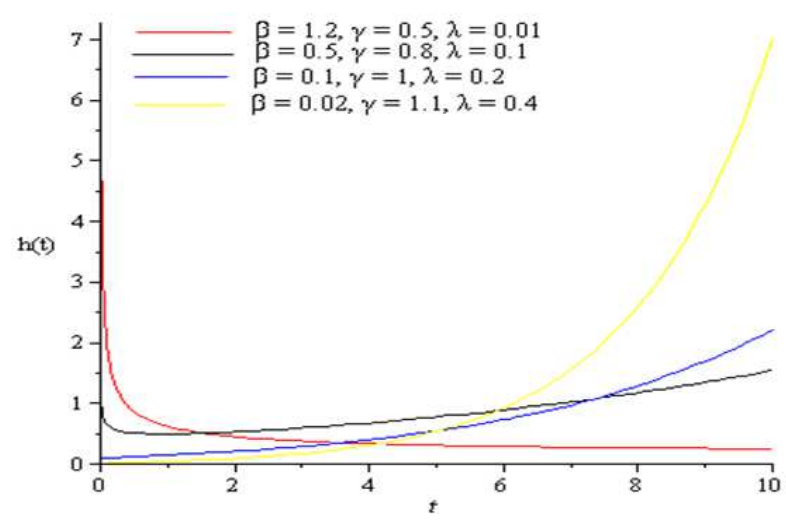

Fig. 2. Different shapes of HRF versus time 


\section{Random Number Generation}

Using the method of inversion we can generate random numbers from the modified Weibull distribution as follows:

$$
F(t)=1-e^{-\beta t^{r} e^{\lambda t}}=u
$$

where, $u \sim$ uniform $(0,1)$. After simple calculation this yields:

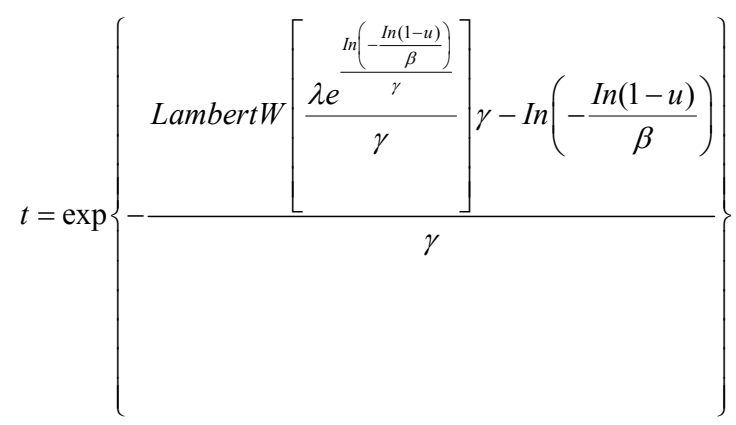

We can use the previous relation to generate random numbers when the parameters $\beta, \gamma$ and $\lambda$ are known where Lambert $W$ is a function that satisfies the following relation:

$$
\operatorname{Lambert} W(x) * \exp (\text { Lambert } W(x))=x
$$

\section{Parameters Estimation}

The maximum likelihood method is used to find point estimators for the parameters of the modified Weibull distribution as follows:

Let $T_{1}, T_{2}, \ldots, T_{n}$ be a sample of size $\mathrm{n}$ from a modified Weibull distribution. Then the likelihood function is given by:

$$
L(\beta, \gamma, \lambda)=\prod_{i=1}^{n} f\left(t_{i}\right)=\prod_{i=1}^{n} \beta\left(\gamma+\lambda t_{i}\right) t_{i}^{\gamma-1} e^{\lambda t_{i}} e^{-\beta t_{i}^{\gamma} e^{\lambda t_{i}}}
$$

Take the logarithm of the likelihood function:

$$
\log L(\beta, \gamma, \lambda)=\sum_{i=1}^{n}\left[\begin{array}{l}
\log (\beta)+\log \left(\gamma+\lambda t_{i}\right) \\
+(\gamma-1) \log \left(t_{i}\right)+\lambda t_{i}
\end{array}\right]-\beta \sum_{i=1}^{n} t_{i}^{\gamma} e^{\lambda t_{i}}
$$

The first partial derivatives with respect to $\beta, \gamma$ and $\lambda$ are derived as follows:

$$
\begin{aligned}
\frac{\partial \log L(\beta, \gamma, \lambda)}{\partial \beta} & =\sum_{i=1}^{n} \frac{1}{\beta}-\sum_{i=1}^{n} t_{i}^{\gamma} e^{\lambda t_{i}}=\frac{n}{\beta}-\sum_{i=1}^{n} t_{i}^{\gamma} e^{\lambda t_{i}} \\
\frac{\partial \log L(\beta, \gamma, \lambda)}{\partial \gamma} & =\sum_{i=1}^{n} \frac{1}{\left(\gamma+\lambda t_{i}\right)}+\sum_{i=1}^{n} \log \left(t_{i}\right) \\
& -\beta \sum_{i=1}^{n} t_{i}^{\gamma} \log \left(t_{i}\right) e^{\lambda t_{i}}
\end{aligned}
$$

$\frac{\partial \log L(\beta, \gamma, \lambda)}{\partial \lambda}=\sum_{i=1}^{n} \frac{t_{i}}{\left(\gamma+\lambda t_{i}\right)}+\sum_{i=1}^{n} t_{i}-\beta \sum_{i=1}^{n} t_{i}^{\gamma+1} e^{\lambda t_{i}}$

In order to obtain the point estimators $\hat{\theta}=(\hat{\beta}, \hat{\gamma}, \hat{\lambda})$ for the parameters $\theta=(\beta, \gamma, \lambda)$, the previous system of nonlinear equations can be solved numerically after equating them to zero.

Second derivatives of the logarithm of the likelihood function are derived and the results are:

$$
\begin{aligned}
& \frac{\partial^{2} \log L(\beta, \gamma, \lambda)}{\partial \beta^{2}}=-\frac{n}{\beta^{2}} \\
& \frac{\partial^{2} \log L(\beta, \gamma, \lambda)}{\partial \gamma^{2}}=-\sum_{i=1}^{n} \frac{1}{\left(\gamma+\lambda t_{i}\right)^{2}}-\beta \sum_{i=1}^{n} t_{i}^{\gamma}\left[\log \left(t_{i}\right)\right]^{2} e^{\lambda t_{i}} \\
& \frac{\partial^{2} \log L(\beta, \gamma, \lambda)}{\partial \lambda^{2}}=-\sum_{i=1}^{n} \frac{t_{i}^{2}}{\left(\gamma+\lambda t_{i}\right)^{2}}-\beta \sum_{i=1}^{n} t_{i}^{\gamma+2} e^{\lambda t_{i}} \\
& \frac{\partial^{2} \log L(\beta, \gamma, \lambda)}{\partial \gamma \partial \beta}=\frac{\partial^{2} \log L(\beta, \gamma, \lambda)}{\partial \beta \partial \gamma}=-\sum_{i=1}^{n} t_{i}^{\gamma} \log \left(t_{i}\right) e^{\lambda t_{i}} \\
& \frac{\partial^{2} \log L(\beta, \gamma, \lambda)}{\partial \lambda \partial \beta}=\frac{\partial^{2} \log L(\beta, \gamma, \lambda)}{\partial \beta \partial \lambda}=-\sum_{i=1}^{n} t_{i}^{\gamma+1} e^{\lambda t_{i}} \\
& \frac{\partial^{2} \log L(\beta, \gamma, \lambda)}{\partial \lambda \partial \gamma}=\frac{\partial^{2} \log L(\beta, \gamma, \lambda)}{\partial \gamma \partial \lambda} \\
& =-\sum_{i=1}^{n} \frac{t_{i}}{\left(\gamma+\lambda t_{i}\right)^{2}}-\beta \sum_{i=1}^{n} t_{i}^{\gamma+1} \log \left(t_{i}\right) e^{\lambda t_{i}}
\end{aligned}
$$

The observed information matrix is defined as:

$$
I(\theta)=-\left(\begin{array}{lll}
\frac{\partial^{2} \log L(\beta, \gamma, \lambda)}{\partial \beta^{2}} & \frac{\partial^{2} \log L(\beta, \gamma, \lambda)}{\partial \gamma \partial \beta} & \frac{\partial^{2} \log L(\beta, \gamma, \lambda)}{\partial \lambda \partial \beta} \\
\frac{\partial^{2} \log L(\beta, \gamma, \lambda)}{\partial \beta \partial \gamma} & \frac{\partial^{2} \log L(\beta, \gamma, \lambda)}{\partial \gamma^{2}} & \frac{\partial^{2} \log L(\beta, \gamma, \lambda)}{\partial \lambda \partial \gamma} \\
\frac{\partial^{2} \log L(\beta, \gamma, \lambda)}{\partial \beta \partial \lambda} & \frac{\partial^{2} \log L(\beta, \gamma, \lambda)}{\partial \gamma \partial \lambda} & \frac{\partial^{2} \log L(\beta, \gamma, \lambda)}{\partial \lambda^{2}}
\end{array}\right)
$$

In order to compute the standard error and asymptotic confidence interval the usual large sample approximation is used in which the maximum likelihood estimators of $\theta$ can be treated as approximately normal. Hence as $n \rightarrow \infty$, the asymptotic distribution of the $\operatorname{MLE}(\hat{\beta}, \hat{\gamma}, \hat{\lambda})$ will be given by:

$$
\left(\begin{array}{l}
\hat{\beta} \\
\hat{\gamma} \\
\hat{\lambda}
\end{array}\right) \sim N\left[\left(\begin{array}{l}
\beta \\
\gamma \\
\lambda
\end{array}\right),\left(\begin{array}{lll}
\hat{V}_{11} & \hat{V}_{12} & \hat{V}_{13} \\
\hat{V}_{21} & \hat{V}_{22} & \hat{V}_{23} \\
\hat{V}_{31} & \hat{V}_{32} & \hat{V}_{33}
\end{array}\right)\right]
$$

where, $\hat{V}_{i j}=\left.V_{i j}\right|_{\theta=\hat{\theta}}$ and: 


$$
\left(\begin{array}{lll}
V_{11} & V_{12} & V_{13} \\
V_{21} & V_{22} & V_{23} \\
V_{31} & V_{32} & V_{33}
\end{array}\right)=I^{-1}(\theta)
$$

is the approximate variance covariance matrix and $\Gamma^{-1}(\theta)$ is the inverse of the observed information matrix. An Approximate $100(1-\alpha) \%$ two sided confidence intervals for $\beta, \gamma$ and $\lambda$ are, respectively, given by:

$$
\hat{\beta} \pm z_{\alpha / 2} \sqrt{\hat{V}_{11}}, \hat{\gamma} \pm z_{\alpha / 2} \sqrt{\hat{V}_{22}}, \hat{\lambda} \pm z_{\alpha / 2} \sqrt{\hat{V}_{33}}
$$

\section{Fuzzy Parameters}

Now let us suppose that the parameters of the modified Weibull distribution are fuzzy. A fuzzy set is defined as a function that maps elements in a domain of concern to their membership value in a set and this function is called membership function. The membership function of a fuzzy set $A$ is denoted as $\mu_{A}$ and membership value of $x$ in $A$ is denoted as $\mu_{A}(x)$. The domain of membership function is called the universe of discourse.

\section{Definition 1}

Dijkman et al. (1983) defined fuzzy number as a generalization of a regular, real number in the sense that it does not refer to one single value but rather to a connected set of possible values, where each possible value has its own weight between 0 and 1 .

\section{Definition 2}

A membership function $\mu_{A}(x)$ of a fuzzy set $\mathrm{A}$ is a function $\mu_{A}: X \rightarrow[0,1]$ such that every element $x$ in $X$ has membership degree $\mu_{A}(x) \in[0,1]$.

We suppose that the type of the membership functions is the triangular one. In general, the triangular membership function is defined as follows.

\section{Definition 3}

A triangular membership function is determined by three numbers $\{a, b, c\}$ where $a$ is the lowest value, $b$ is the nominal value and $c$ is the maximum value. The triangular membership function is given by:

$$
\operatorname{triangle}(x ; a, b, c)=\left[\begin{array}{cc}
0, & x<a \\
(x-a) /(b-a), & a \leq x \leq b \\
(c-x) /(c-b), & b \leq x \leq c^{\prime} \\
0, & x>c
\end{array}\right]
$$

where, the parameters $\{a, b, c\}$, with $(a<b<c)$, determine the $x$ coordinates of the three corners of the underlying triangular membership function.

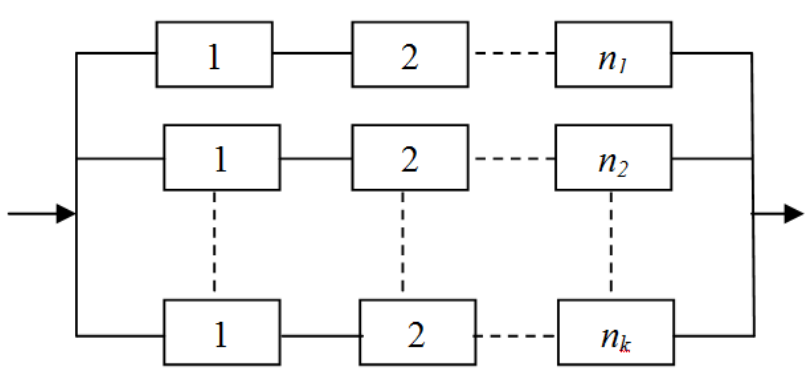

Fig. 3. A series-parallel system

\section{Original System}

We consider a series-parallel system consisted of $k$ subsystems connected in parallel and each subsystem consists of $n_{i}$ units connected in series for $i=1,2, \ldots, k$. The system will operate successfully when at least one subsystem is up (see Fig. 3).

Let $R_{i j}(t)$ be the reliability function of the unit $j(j=$ $1,2, \ldots, n_{i}$ ) of a subsystem $i, i=1,2, \ldots, k$. Hence, the reliability function of the system will be given by:

$$
\begin{aligned}
& R(t)=1-\prod_{i=1}^{k}\left[1-\prod_{j=1}^{n_{i}} R_{i j}(t)\right] \\
& R(t)=1-\prod_{i=1}^{k}\left[1-\prod_{j=1}^{n_{i}} e^{\left.-\beta_{i j} t^{\gamma_{i j}} e^{\lambda_{i j} t}\right]}\right. \\
& R(t)=1-\prod_{i=1}^{k}\left[1-\exp \left\{-\sum_{j=1}^{n_{i}} \beta_{i j} t^{\gamma_{i j}} e^{\lambda_{i j} t}\right\}\right]
\end{aligned}
$$

If all units of the system are identical, then the reliability function will be given by:

$$
R(t)=1-\prod_{i=1}^{k}\left[1-\exp \left\{-n_{i} \beta t^{\gamma} e^{\lambda t}\right\}\right]
$$

\section{Reduction Method}

It is assumed in the reduction method that the system design can be improved by reducing the failure rates of a set of its components by a factor $\rho$ such that $0<\rho<1$ (Sarhan et al., 2004; Sarhan, 2009). We suppose that the failure rates of a set $A_{i}$ of the units of each subsystem are decreased by multiplying by a factor $\rho$ and hence system reliability function is obtained as follows:

$$
\begin{aligned}
& R(t)=1-\prod_{i=1}^{k}\left[1-\prod_{j \in A_{i}} R_{i j, \rho}(t) \prod_{j \notin A_{i}} R_{i j}(t)\right] \\
& R(t)=1-\prod_{i=1}^{k}\left[1-\exp \left\{-\sum_{j \in A_{i}} \rho \beta_{i j} t^{\gamma_{i j}} e^{\lambda_{i j} t}+\sum_{j \notin A_{i}} \beta_{i j} t^{\gamma_{i j}} e^{\lambda_{j i} t}\right\}\right]
\end{aligned}
$$

where: 


$$
A_{i} \subseteq\left\{1,2, . ., n_{i}\right\}, i=1,2, \ldots, k
$$

\section{Cold Standby Duplication Method}

In this method, we suppose that a set $C_{i}$ of components of each subsystem are duplicated with an identical cold standby unit. The reliability function of the improved system is obtained as follows:

$$
\begin{aligned}
& R_{c}(t)=1-\prod_{i=1}^{k}\left[1-\prod_{j \in C_{i}} R_{i j, c}(t) \prod_{j \notin C_{i}} R_{i j}(t)\right] \\
& R_{c}(t)=1-\prod_{i=1}^{k}\left[1-\prod_{j \in C_{i}}\left(1+\beta_{i j} t^{\gamma_{i j}} e^{\lambda_{i j} t}\right) e^{-\beta_{i j} t^{\gamma_{i j}} e^{\lambda_{j i} t}} \prod_{j \notin C_{i}} e^{\left.-\beta_{i j} t^{\gamma_{i j}} e^{\lambda_{i j} t}\right]}\right. \\
& R_{c}(t)=1-\prod_{i=1}^{k}\left[1-\exp \left\{-\sum_{j=1}^{n_{i}} \beta_{i j} t^{\gamma_{i j}} e^{\lambda_{i j} t}\right\} \prod_{j \in C_{i}}\left(1+\beta_{i j} t^{\gamma_{i j}} e^{\lambda_{i j} t}\right)\right]
\end{aligned}
$$

where:

$$
C_{i} \subseteq\left\{1,2, . ., n_{i}\right\}, i=1,2, \ldots, k
$$

\section{Hot Standby Duplication Method}

In this method, we suppose that a set of $D_{i}$ components of each subsystem are duplicated with a hot standby unit. The reliability function of the improved system is obtained as follows:

$$
\begin{aligned}
& R_{h}(t)=1-\prod_{i=1}^{k}\left[1-\prod_{j \in D_{i}} R_{i j, h}(t) \prod_{j \notin D_{i}} R_{i j}(t)\right]
\end{aligned}
$$

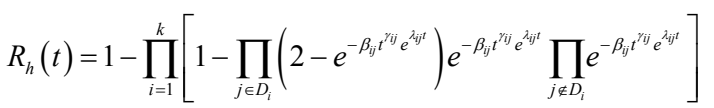

$$
\begin{aligned}
& R_{h}(t)=1-\prod_{i=1}^{k}\left[1-\exp \left\{-\sum_{j=1}^{n_{i}} \beta_{i j} t^{\gamma_{i j}} e^{\lambda_{i j} t}\right\} \prod_{j \in D_{i}}\left(2-e^{-\beta_{i j} t^{\gamma_{j}} e^{\lambda_{j i} t}}\right)\right]
\end{aligned}
$$

Where:

$$
D_{i} \subseteq\left\{1,2, . ., n_{i}\right\}, i=1,2, \ldots, k
$$

\section{Procedure}

In this section, we introduce a procedure to illustrate how the reliability of the system is computed:

Step 1: Generate a random sample from the modified Weibull distribution at fixed values of the parameters $(\beta, \gamma, \lambda)$ by using relation (4).

Step 2: Substituting in a set of equations (7)-(9) and then equating them to zero

Step 3: Obtain the MLE $(\hat{\beta}, \hat{\gamma}, \hat{\lambda})$ by solving the resultant system of equations numerically

Step 4: Constructing the observed information matrix and compute its inverse

Step 5: Calculate the confidence intervals for the parameters $(\beta, \gamma, \lambda)$ at a level of significance $a$
Step 6: Calculate the intervals for the fuzzy parameters by substituting in the following relation:

$$
\left[\hat{\theta}_{L}, \hat{\theta}_{U}\right]=\left[\begin{array}{c}
L+(\alpha-c u t)(M-L), U \\
+(\alpha-c u t)(U-M)
\end{array}\right] \text { for } \theta=(\beta, \gamma, \lambda)
$$

where, $a-c u t=\{0,0.1,0.2, \ldots, 1\}$ and $M$ is the point estimator of $\theta$ and $[L, U]$ is the confidence interval limits of $\theta$.

Step 7: Substituting in equations (10), (12), (13) and (14) to obtain intervals for the fuzzy reliability function of the original system and the improved systems, respectively.

\section{Numerical Study}

Let us consider a series-parallel system consists of two subsystems connected in parallel. The first subsystem consists of two units connected in series and the second one consists of one unit. Suppose that all units are identical. We will apply our procedure to obtain the limits for the fuzzy reliability of the system. The reliability function of the original system will be given by:

$$
R(t)=1-\left(1-\exp \left(-2 \beta t^{\gamma} e^{\lambda t}\right)\right)\left(1-\exp \left(-\beta t^{\gamma} e^{\lambda t}\right)\right)
$$

Now, we will generate a random sample with size $\mathrm{n}=$ 30 at $(\beta, \gamma, \lambda)=(0.1,0.7,0.3)$ and the result is:

$\begin{array}{lllll}4.9 & 3.9 & 5.2 & 3.9 & 1.1 \\ 1.8 & 2.7 & 1.0 & 5.4 & 0.9 \\ 0.6 & 5.6 & 6.0 & 1.0 & 3.3 \\ 3.6 & 0.1 & 5.1 & 1.3 & 5.3 \\ 7.5 & 5.1 & 3.4 & 3.8 & 6.0 \\ 5.6 & 3.1 & 2.6 & 0.6 & 0.3\end{array}$

Substituting in a set of equations (7)-(9) and then equating them to zero. One can obtain the $\operatorname{MLE}(\hat{\beta}, \hat{\gamma}, \hat{\lambda})$ by solving the resultant system of equations numerically and the values are:

$$
(\hat{\beta}, \hat{\gamma}, \hat{\lambda})=(0.119,0.763,0.261)=(L, M, U)
$$

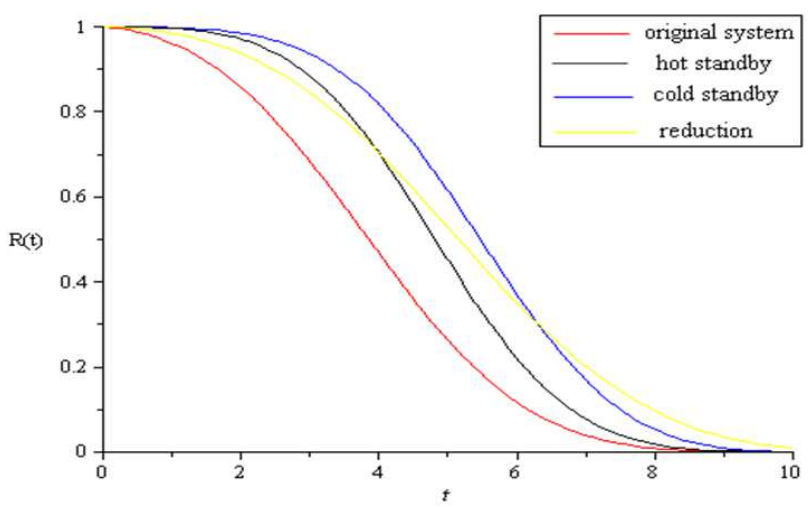

Fig. 4. Comparison of the reliabilities of the original system and different improved systems 
In Fig. (4), we can observe that the reliability of the original system is improved by different ways. Cold standby method is better than hot duplication method and reduction method has different values which improves the system reliability.

We construct the observed information matrix and the result is:

$$
I(\theta)=\left(\begin{array}{ccc}
2107.177 & 391.958 & 1280.519 \\
391.958 & 94.728 & 287.520 \\
1280.519 & 287.520 & 959.924
\end{array}\right)
$$

Taking the inverse of this matrix yields the following matrix:

$$
I^{-1}(\theta)=\left(\begin{array}{ccc}
0.00255 & -0.00250 & 0.00266 \\
-0.00250 & 0.11860 & -0.03218 \\
-0.00266 & -0.03218 & 0.014237
\end{array}\right)
$$

Calculate the $95 \%$ confidence intervals for the parameters $(\beta, \gamma, \lambda)$ and the results are:

$$
\begin{aligned}
& \text { Confidence interval for } \beta=[0.020,0.217] \\
& \text { Confidence interval for } \gamma=[0.088,1.437] \\
& \text { Confidence interval for } \lambda=[0.027,0.494]
\end{aligned}
$$

Now, we consider that the parameters $\beta, \gamma$ and $\lambda$ are triangular fuzzy numbers then calculate the intervals for them by using in relation (15) and the results are shown in Table 1.

The intervals for the fuzzy reliability function of the original system will be calculated from the following relation:

$$
\left[\tilde{R}_{L}(t), \tilde{R}_{U}(t)\right]=\left[\begin{array}{l}
1-\left(1-\exp \left(-2 \tilde{\beta}_{L} t^{\tilde{\gamma}_{L}} e^{\tilde{\lambda}_{L} t}\right)\right) \\
\left(1-\exp \left(-\tilde{\beta}_{L} t^{\tilde{t}_{L}} e^{\tilde{\lambda}_{L} t}\right)\right) \\
1-\left(1-\exp \left(-2 \tilde{\beta}_{U} \tilde{t}^{\tilde{t}_{U}} e^{\tilde{\lambda}_{L} t}\right)\right) \\
\left(1-\exp \left(-\tilde{\beta}_{U} t^{\tilde{\gamma}_{U}} e^{{\tilde{\lambda_{U}}}_{U} t}\right)\right)
\end{array}\right]
$$

Substituting the values of the fuzzy parameters from Table 1 in the above relation, we obtain the intervals for the fuzzy reliability function of the original system at a fixed time $(t=2)$ and the results are shown in Table 2 .

\begin{tabular}{|c|c|c|c|}
\hline$\alpha-c u t$ & {$\left[\tilde{\beta}_{L}, \tilde{\beta}_{U}\right]$} & {$\left[\tilde{\gamma}_{L}, \tilde{\gamma}_{U}\right]$} & {$\left[\tilde{\lambda}_{L}, \tilde{\lambda}_{U}\right]$} \\
\hline 0.0 & {$[0.020,0.217]$} & {$[0.088,1.437]$} & {$[0.027,0.494]$} \\
\hline 0.1 & {$[0.029,0.266]$} & {$[0.155,1.504]$} & {$[0.050,0.517]$} \\
\hline 0.2 & {$[0.039,0.236]$} & {$[0.223,1.571]$} & {$[0.073,0.540]$} \\
\hline 0.3 & {$[0.049,0.246]$} & {$[0.290,1.639]$} & {$[0.097,0.563]$} \\
\hline 0.4 & {$[0.059,0.256]$} & {$[0.358,1.706]$} & {$[0.120,0.587]$} \\
\hline 0.5 & {$[0.069,0.266]$} & {$[0.425,1.774]$} & {$[0.144,0.610]$} \\
\hline 0.6 & {$[0.079,0.275]$} & {$[0.493,1.841]$} & {$[0.167,0.633]$} \\
\hline 0.7 & {$[0.089,0.285]$} & {$[0.560,1.908]$} & {$[0.190,0.657]$} \\
\hline 0.8 & {$[0.099,0.295]$} & {$[0.628,1.976]$} & {$[0.214,0.680]$} \\
\hline 0.9 & {$[0.109,0.305]$} & {$[0.695,2.043]$} & {$[0.237,0.703]$} \\
\hline 1.0 & {$[0.119,0.315]$} & {$[0.763,2.111]$} & {$[0.261,0.727]$} \\
\hline
\end{tabular}
Also, we obtain the intervals for the fuzzy reliability functions of the improved systems using reduction, cold standby and hot duplication methods at a fixed time $(t=2)$ and the results are shown in Tables 3-5, respectively.

Table 1. Calculations of the intervals for fuzzy parameters

Table 2. Calculations of the intervals for the fuzzy reliability function at time $t=2$

\begin{tabular}{ll}
\hline$a-c u t$ & {$\left[\tilde{R}_{L}, \tilde{R}_{U}\right]$} \\
\hline 0.0 & {$[0.240,0.999]$} \\
0.1 & {$[0.187,0.997]$} \\
0.2 & {$[0.140,0.994]$} \\
0.3 & {$[0.102,0.990]$} \\
0.4 & {$[0.083,0.983]$} \\
0.5 & {$[0.047,0.974]$} \\
0.6 & {$[0.031,0.961]$} \\
0.7 & {$[0.019,0.944]$} \\
0.8 & {$[0.010,0.921]$} \\
0.9 & {$[0.005,0.893]$} \\
1.0 & {$[0.002,0.857]$} \\
\hline
\end{tabular}

Table 3. Calculations of intervals of fuzzy reliability function using reduction method at time $t=2$

\begin{tabular}{llcccc}
\hline$\alpha-c u t$ & {$\left[\tilde{R}_{p}^{\{1,0\}}{ }_{L} \tilde{R}_{p}^{\{1,0\}}{ }_{U}\right]$} & {$\left[\tilde{R}_{p}^{\{0,1\}}{ }_{L}{ }^{\prime} \tilde{R}_{p}^{\{0,1\}}{ }_{U}\right]$} & {$\left[\tilde{R}_{p}^{\{2,0\}}{ }_{L}, \tilde{R}_{p}^{\{2,0\}}{ }_{U}\right]$} & {$\left[\tilde{R}_{p}^{\{1,1\}}{ }_{L}, \tilde{R}_{p}^{\{1,1\}}{ }_{U}\right]$} & {$\left[\tilde{R}_{p}^{\{2,1\}}{ }_{{ }^{\prime}} \tilde{R}_{p}^{\{2,1\}}{ }_{U}\right]$} \\
\hline 0.0 & {$[0.280,0.999]$} & {$[0.477,0.999]$} & {$[0.370,0.999]$} & {$[0.505,0.999]$} & {$[0.566,0.999]$} \\
0.1 & {$[0.220,0.998]$} & {$[0.422,0.998]$} & {$[0.302,0.998]$} & {$[0.445,0.999]$} & {$[0.503,0.999]$} \\
0.2 & {$[0.166,0.996]$} & {$[0.366,0.997]$} & {$[0.237,0.997]$} & {$[0.385,0.998]$} & {$[0.437,0.998]$} \\
0.3 & {$[0.120,0.992]$} & {$[0.313,0.995]$} & {$[0.179,0.995]$} & {$[0.326,0.996]$} & {$[0.372,0.997]$} \\
0.4 & {$[0.083,0.987]$} & {$[0.262,0.991]$} & {$[0.129,0.991]$} & {$[0.271,0.993]$} & {$[0.308,0.995]$} \\
0.5 & {$[0.055,0.980]$} & {$[0.215,0.986]$} & {$[0.089,0.986]$} & {$[0.221,0.989]$} & {$[0.250,0.993]$} \\
0.6 & {$[0.035,0.970]$} & {$[0.175,0.980]$} & {$[0.059,0.979]$} & {$[0.178,0.984]$} & {$[0.199,0.989]$} \\
0.7 & {$[0.021,0.956]$} & {$[0.137,0.970]$} & {$[0.037,0.969]$} & {$[0.138,0.977]$} & {$[0.152,0.984]$} \\
0.8 & {$[0.011,0.937]$} & {$[0.104,0.958]$} & {$[0.021,0.956]$} & {$[0.105,0.967]$} & {$[0.114,0.976]$} \\
0.9 & {$[0.006,0.914]$} & {$[0.077,0.942]$} & {$[0.011,0.939]$} & {$[0.077,0.954]$} & {$[0.082,0.967]$} \\
1.0 & {$[0.003,0.884]$} & {$[0.054,0.922]$} & {$[0.005,0.916]$} & {$[0.054,0.937]$} & {$[0.057,0.954]$} \\
\hline
\end{tabular}


Table 4. Calculations of intervals for fuzzy reliability function using cold standby method at time $t=2$

\begin{tabular}{llcccc}
\hline$a-c u t$ & {$\left[\tilde{R}_{C}^{\{1,0\}}{ }_{L} \tilde{R}_{C}^{\{1,0\}}{ }_{U}\right]$} & {$\left[\tilde{R}_{C}^{\{0,1\}}{ }_{L} \tilde{R}_{C}^{\{0,1\}}{ }_{U}\right]$} & {$\left[\tilde{R}_{C}^{\{2,0\}}{ }_{L}, \tilde{R}_{C}^{\{2,0\}}{ }_{U}\right]$} & {$\left[\tilde{R}_{C}^{\{1,1\}}{ }{ } \tilde{R}_{C}^{\{1,1\}}{ }_{U}\right]$} & {$\left[\tilde{R}_{C}^{\{2,1\}}{ }_{L}, \tilde{R}_{C}^{\{2,1\}}{ }_{U}\right]$} \\
\hline 0.0 & {$[0.293,0.999]$} & {$[0.551,0.999]$} & {$[0.431,0.999]$} & {$[0.583,0.999]$} & {$[0.664,0.999]$} \\
0.1 & {$[0.228,0.998]$} & {$[0.476,0.999]$} & {$[0.343,0.999]$} & {$[0.503,0.999]$} & {$[0.576,0.999]$} \\
0.2 & {$[0.169,0.997]$} & {$[0.398,0.999]$} & {$[0.258,0.999]$} & {$[0.418,0.999]$} & {$[0.481,0.999]$} \\
0.3 & {$[0.121,0.994]$} & {$[0.322,0.999]$} & {$[0.185,0.999]$} & {$[0.337,0.999]$} & {$[0.385,0.999]$} \\
0.4 & {$[0.082,0.991]$} & {$[0.251,0.999]$} & {$[0.124,0.999]$} & {$[0.260,0.999]$} & {$[0.294,0.999]$} \\
0.5 & {$[0.054,0.985]$} & {$[0.189,0.998]$} & {$[0.079,0.998]$} & {$[0.194,0.999]$} & {$[0.215,0.999]$} \\
0.6 & {$[0.034,0.977]$} & {$[0.137,0.997]$} & {$[0.048,0.996]$} & {$[0.140,0.998]$} & {$[0.152,0.999]$} \\
0.7 & {$[0.020,0.967]$} & {$[0.093,0.994]$} & {$[0.027,0.994]$} & {$[0.094,0.996]$} & {$[0.100,0.999]$} \\
0.8 & {$[0.011,0.952]$} & {$[0.060,0.991]$} & {$[0.014,0.990]$} & {$[0.060,0.994]$} & {$[0.063,0.998]$} \\
0.9 & {$[0.006,0.932]$} & {$[0.036,0.985]$} & {$[0.007,0.983]$} & {$[0.036,0.990]$} & {$[0.037,0.997]$} \\
1.0 & {$[0.003,0.907]$} & {$[0.020,0.977]$} & {$[0.003,0.973]$} & {$[0.020,0.985]$} & {$[0.020,0.995]$} \\
\hline
\end{tabular}

Table 5. Calculations of intervals for fuzzy reliability using hot duplication method at time $t=2$

\begin{tabular}{llcccc}
\hline$\alpha$-cut & {$\left[\tilde{R}_{H}^{\{1,0\}}{ }_{L} \tilde{R}_{H}^{\{1,0\}}\right]$} & {$\left[\tilde{R}_{H}^{\{0,1\}}{ }_{L} \tilde{R}_{H}^{\{0,1\}}\right]$} & {$\left[\tilde{R}_{H}^{\{2,0\}}{ }_{L}, \tilde{R}_{H}^{\{2,0\}}{ }_{U}\right]$} & {$\left[\tilde{R}_{H}^{\{1,1\}}{ }_{L} \tilde{R}_{H}^{\{1,1\}}{ }_{U}\right]$} & {$\left[\tilde{R}_{H}^{\{2,1\}}{ }_{{ }} \tilde{R}_{H}^{\{2,1\}}{ }_{U}\right]$} \\
\hline 0.0 & {$[0.267,0.999]$} & {$[0.396,0.999]$} & {$[0.315,0.999]$} & {$[0.418,0.999]$} & {$[0.456,0.999]$} \\
0.1 & {$[0.206,0.998]$} & {$[0.321,0.999]$} & {$[0.241,0.999]$} & {$[0.337,0.999]$} & {$[0.366,0.999]$} \\
0.2 & {$[0.153,0.997]$} & {$[0.249,0.999]$} & {$[0.176,0.999]$} & {$[0.260,0.999]$} & {$[0.280,0.999]$} \\
0.3 & {$[0.109,0.994]$} & {$[0.186,0.999]$} & {$[0.123,0.999]$} & {$[0.193,0.999]$} & {$[0.205,0.999]$} \\
0.4 & {$[0.075,0.990]$} & {$[0.133,0.998]$} & {$[0.082,0.998]$} & {$[0.137,0.999]$} & {$[0.144,0.999]$} \\
0.5 & {$[0.049,0.985]$} & {$[0.091,0.997]$} & {$[0.054,0.996]$} & {$[0.093,0.998]$} & {$[0.097,0.999]$} \\
0.6 & {$[0.032,0.976]$} & {$[0.060,0.994]$} & {$[0.033,0.994]$} & {$[0.061,0.996]$} & {$[0.063,0.999]$} \\
0.7 & {$[0.019,0.965]$} & {$[0.037,0.990]$} & {$[0.020,0.989]$} & {$[0.037,0.993]$} & {$[0.038,0.998]$} \\
0.8 & {$[0.011,0.948]$} & {$[0.021,0.983]$} & {$[0.011,0.982]$} & {$[0.021,0.989]$} & {$[0.022,0.996]$} \\
0.9 & {$[0.005,0.927]$} & {$[0.011,0.973]$} & {$[0.006,0.970]$} & {$[0.011,0.982]$} & {$[0.011,0.992]$} \\
1.0 & {$[0.002,0.899]$} & {$[0.005,0.958]$} & {$[0.002,0.953]$} & {$[0.005,0.971]$} & {$[0.005,0.986]$} \\
\hline
\end{tabular}

\section{Conclusion}

In this paper, we analyzed a series-parallel system consisted of independent and non-identical components when the lifetimes of the components follow modified Weibull distribution. In literature, the parameters of the lifetime distribution were considered to be known. However, exact values of the parameters of any distribution are often unknown. Here, the parameters of the modified Weibull distribution were considered to be fuzzy which means that they are not longer treated as fixed numbers. Reduction method was used to improve the reliability of the original system. Also, cold and hot duplication methods were introduced to increase system reliability. A procedure was added to show how the intervals for fuzzy reliability function are computed. Numerical study was proposed to compare between different methods. The values obtained in Tables 4 and 5 show that cold standby method is better than hot duplication method for improving system reliability.

As future work, a study of a system assuming that its components are dependent can be treated. Also, increasing the number of standby units can be used to increase systems reliability. New distributions can be used to model the lifetimes of the components of a system.

\section{Acknowledgement}

I would thank the reviwers for their comments which improved my paper.

\section{Funding Information}

There is no funding source for this article.

\section{Ethics}

This article is original.

\section{References}

Chen, S.M., 1994. Fuzzy system reliability analysis using fuzzy number arithmetic operations. Fuzzy Sets Syst., 64: 31-38.

DOI: 10.1016/0165-0114(94)90004-3

Chen, S.M., 1996. New method for fuzzy system reliability analysis, cybernetics and systems. Int. J., 27: 385-401. DOI: 10.1080/019697296126480

Cheng, C.H. and D.L. Mon, 1993. Fuzzy system reliability analysis by interval of confidence. Fuzzy Sets Syst., 56: 29-35.

DOI: 10.1016/0165-0114(93)90182-H 
Dijkman, J.G., Haeringen, H. van and S.J. Lange, 1983. Fuzzy numbers. J. Mathematical Analysis Appli., 92: 301-341. DOI: 10.1016/0022-247X(83)90253-6

El-Damcese, M.A. and N.S. Temraz, 2015. Analysis of availability and reliability of k-out-of-n: F model with fuzzy rates. Int. J. Computational Sci. Eng.

El-Damcese, M.A., 2009. Reliability equivalence factors of a series-parallel system in Weibull distribution. Int. J. Mathematical Forum, 4: 941-951.

Ezzati, G. and A. Rasouli, 2015. Evaluating system reliability using linear-exponential distribution function. Int. J. Advanced Statistics Probability, 3: 15-24. DOI: 10.14419/ijasp.v3i1.3927

Hoyland, A. and M. Rausand, 2004. System Reliability Theory: Models and Statistical Methods, Wiley and Sons, Canada.

Khan, A.H. and T.R. Jan, 2015. Reliability evaluation of engineering system using modified Weibull distribution. Res. J. Math. Statistical Sci., 3: 1-8.

Lai, C.D., M. Xie and D.N.P. Murthy, 2003. Modified weibull model. IEEE Trans. Reliability, 52: 33-37. DOI: $10.1109 /$ TR.2002.805788
Mustafa, A., 2009. Reliability equivalence factors for some systems with mixture Weibull failure rates. African J. Mathematics Comput. Sci. Res., 2: 6-13.

Novák, V., I. Perfilieva and J. Močkoř, 1999. Mathematical principles of fuzzy logic. Kluwer Academic Publishers, London.

Poǵany, T.K., V. Tomas and M. Tudor, 2013. Hot duplication versus survivor equivalence in GammaWeibull distribution. J. Statistics Applications Probability, 1: 1-10. DOI: 10.12785/jsap/020101

Sarhan, A.M., 2009. Reliability equivalence factors of a general series-parallel system. J. Reliability Eng. Syst. Safety, 94: 229-236.

DOI: $10.1016 /$ j.ress.2008.02.021

Sarhan, A.M., A.S. Al-Ruzaiza and I.A. El-Gohary, 2004. Reliability equivalence of a series-parallel system. J. Applied Math. Computation, 154: 257-277. DOI: 10.1016/S0096-3003(03)00709-4

Zadeh, L., 1965. Fuzzy sets. J. Information Control, 8: 338-353. DOI: 10.1016/S0019-9958(65)90241-X 